\title{
Factors Associated with Vitamin D Deficiency in European Adolescents: The HELENA Study
}

\author{
Jara VAltueÑA ${ }^{1,2}$, Marcela GonZÁLEZ-Gross ${ }^{1,3}$, Inge HuYbrechts ${ }^{2}$, Christina BreIDENASseL ${ }^{1,3}$, \\ Marika FERRARI $^{4}$, Theodora MOURATIDOU ${ }^{5}$, Frederic GOTTRAND ${ }^{6}$, Jean DALLONGEVILLE ${ }^{7}$, \\ Elena AZZINI ${ }^{4}$, Isabelle SioEN ${ }^{2,8}$, Sonia GóMEZ-MARTínEZ ${ }^{9}$, Magdalena CUENCA-GARCíA ${ }^{10}$, \\ Mathilde Kersting ${ }^{11}$, Peter SteHLE ${ }^{3}$, Anthony Kafatos ${ }^{12}$, Yannis MAnios ${ }^{13}$, \\ Kurt WIDHALM $^{14}$, Luis A. MORENO ${ }^{5,15}$, on behalf of the HELENA Study Group* \\ ${ }^{1}$ ImFine Research Group, Department of Health and Human Performance, Faculty of Physical Activity and \\ Sport Sciences (INEF), Universidad Politécnica de Madrid, C/Martín Fierro, 7, 28040 Madrid, Spain \\ ${ }^{2}$ Department of Public Health, Faculty of Medicine, Ghent University, Ghent, Belgium \\ ${ }^{3}$ Institut für Ernährungs- und Lebensmittelwissenschaften-Humanernährung, \\ Rheinische Friedrich-Wilhelms Universität Bonn, Germany \\ ${ }^{4}$ National Research Institute on Food and Nutrition, Rome, Italy \\ ${ }^{5}$ Facultad de Ciencias de la Salud, Departamento de Fisiatría y Enfermería, \\ Universidad de Zaragoza, Zaragoza, Spain \\ ${ }^{6}$ Inserm U995, IFR 114, University of Lille 2, Lille, France \\ ${ }^{7}$ Institut Pasteur de Lille, INSERM U744, Lille, France \\ ${ }^{8}$ Research Foundation-Flanders, Brussels, Belgium \\ ${ }^{9}$ Immunonutrition Research Group, Department of Metabolism and Nutrition, Institute of Food Science and \\ Technology and Nutrition (ICTAN), Spanish National Research Council (CSIC), Madrid, Spain \\ ${ }^{10}$ Department of Medical Physiology, School of Medicine, Granada University, Granada, Spain \\ ${ }^{11}$ Research Institute of Child Nutrition Dortmund, Rheinische \\ Friedrich-Wilhelms Universität Bonn, Germany \\ ${ }^{12}$ Preventive Medicine and Nutrition Clinic, University of Crete School of Medicine, Iraclion, Crete, Greece \\ ${ }^{13}$ Department of Nutrition and Dietetics, Harokopio University, Athens, Greece \\ ${ }^{14}$ Department of Pediatrics, Private Medical University, Salzburg, Austria \\ ${ }^{15}$ Grupo de Investigación GENUD: Growth, Exercise, Nutrition and Development
}

(Received November 27, 2012)

\begin{abstract}
Summary Evidence indicates low 25-hydroxyvitamin D [(25(OH)D] concentrations in European adolescents. Identification of potential determinants is therefore essential to guide public health initiatives aiming at optimizing vitamin D status across Europe. The aim of the study was to identify potential influencing factors of $25(\mathrm{OH}) \mathrm{D}$ concentrations in European adolescents aged 12.5 to $17.5 \mathrm{y}$, participating in the multi-centre cross-sectional Healthy Lifestyle in Europe by Nutrition in Adolescence (HELENA) study. A subset of 1,006 participants (46.8\% males) was drawn from the main study. Measures of body composition, biochemical markers, socioeconomic status, dietary intake, physical activity, fitness, sleep time and vitamin D genetic polymorphism (rs1544410) were assessed. Stepwise multivariate linear regression analysis was conducted stratified by gender. In males, linear regression of $25(\mathrm{OH}) \mathrm{D}$, suggested that (1) winter season $(\beta=-0.364 ; p<0.01)$, (2) higher latitudes $(\beta=-0.246 ; p<0.01)$, (3) BMI z-score $(\beta=-0.198 ; p<0.05)$ and $(4)$ retinol concentration $(\beta=0.171 ; p<0.05)$ independently influenced 25(OH)D concentrations. In females, $(1)$ winter season $(\beta=-0.370 ; p<0.01)$, (2) sleep time $(\beta=-0.231 ; p<0.01)$, (3) supplement intake ( $\beta=0.221 ; p<0.05)$, (4) flexibility $(\beta=0.184 ; p<0.05)$, (5) body fat $\%(\beta=0.201$; $p<0.05)$ (6), BMI z-score $(\beta=-0.272 ; p<0.05)$, ( 7 ) higher latitudes $(\beta=-0.219 ; p<0.01)$ and (8) handgrip strength $(\beta=0.206 ; p<0.05)$ independently influenced $25(\mathrm{OH}) \mathrm{D}$ concentrations. Season, latitude, fitness, adiposity, sleep time and micronutrient supplementation were highly related to 25(OH)D concentrations found in European adolescents.
\end{abstract}

Key Words 25(OH)D concentrations, insufficiency, determinants, adolescence, Europe

Adolescence is a critical life-stage period characterised by rapid growth and development. Low circulating 25-hydroxyvitamin D [25(OH)D] concentrations have been negatively associated with obesity and healthy lifestyle habits $(1,2)$. We have previously reported

E-mail: jara.valtuena@upm.es high prevalence (up to $80 \%$ ) of hypovitaminosis D $(<75 \mathrm{nmol} / \mathrm{L})$ in European adolescents participating in the Healthy Lifestyle in Europe by Nutrition in Adolescence (HELENA) study (3). The complexity of vitamin D metabolism poses difficulties in the identification and determination of factors related to vitamin D insufficiency. Its status is largely determined by environmen- 
tal and behavioural factors (4) such as diet, physical activity (PA), physical fitness, geographical location, seasonality and socioeconomic status (5). However, others like age, genetics, body composition (5), and interactions with other liposoluble vitamins could also play an important role (4).

Vitamin D is mainly synthesized in the skin by casual exposure to ultraviolet B (UVB) sunlight. Country-specific geographical locations, and variation in skin production of vitamin $\mathrm{D}$ and latitude differences across Europe, could play the most important role in explaining variance in vitamin D status between northern and southern Europe. There are, however, suggestions indicating that vitamin D production might not compensate for low nutritional intakes (6).

Sun exposure, seasonality and time spent in outdoor activities (including PA or the influence of physical fitness) are also considered to be determinants of vitamin D status in studies during adolescence. Clear associations between sun exposure and seasonality and vitamin D status exist (7), but are inconsistent with PA and fitness levels (8). Obesity, expressed as excess body fat, has an adverse effect on vitamin D status (9), but this relationship in growing children and adolescents is still unclear. In addition, other determinants explaining variation of vitamin D status in European adolescents should be addressed.

The aim of the paper was to determine potential environmental, individual and genetic factors associated with 25(OH)D concentrations in European adolescents aged 12.5 to $17.5 \mathrm{y}$ in order to contribute to optimizing vitamin D status across Europe.

\section{SUBJECTS AND METHODS}

Subjects, recruitment and study design. The HELENACSS (Healthy Lifestyle in Europe by Nutrition in Adolescence) study is a multi-centre cross-sectional study of lifestyle and nutrition among adolescents, from 10 European cities living in nine different countries: Athens and Heraklion (Greece), Dortmund (Germany), Ghent (Belgium), Lille (France), Pecs (Hungary), Rome (Italy), Stockholm (Sweden), Vienna (Austria), and Zaragoza (Spain). Inclusion criteria were being 12.5-17.5 y old, not participating simultaneously in another clinical trial and being free of any acute infection occurring less than $1 \mathrm{wk}$ before inclusion (10). Subjects were recruited by a multi-stage random cluster sampling procedure, using schools as primary sampling units, and classes as secondary sampling units. The size of the sample was calculated according to stratified random sampling with proportional affixation to the size of the strata (sex and age) and minimum variance (Neyman). A confidence level of $95 \%$ and a minimum of \pm 0.3 error for body mass index (BMI) was chosen. On the city level, diversity of the sample with respect to cultural and socioeconomic aspects was achieved by performing a random proportional distribution of all schools taking into account the site (district/zone of the city) and the type of school (public or private) (11). The complete description of the design and implementation of the study has been described elsewhere (11).

Blood sampling was randomly performed in a third of the recruited adolescents due to the low variability of biochemical markers. From a subsample of 1,089 adolescents selected, a subset of 1,006 adolescents $(46 \%$ males) with a mean age of $14.7 \pm 1.2 \mathrm{y}$ completed the blood sampling. This subgroup was recruited between October 2006 and December 2007. The protocol was approved by the Human Research Review Committee of the Universities of Bonn, Lille, Rome, Zaragoza, Athens, Heraklion, Pecs, Ghent and Vienna. The study has been performed following the ethical guidelines of the Declaration of Helsinki 1964 (revision of Edinburgh 2000), the Convention of Oviedo (1997), the Good Clinical Practice, and the legislation about clinical research in humans in each of the participating countries (12). Informed written consent was obtained from subjects and parents or guardians.

Body composition measurements. The complete description of anthropometric measures is published elsewhere (13). Trained personnel conducted the measures in a standardized way. Weight was measured in underwear and without shoes using an electronic scale (Type SECA 861, UK) to the nearest $0.1 \mathrm{~kg}$ and height was measured barefoot in the Frankfort plane with a telescopic height measuring instrument (Type SECA 225 , UK) to the nearest $0.1 \mathrm{~cm}$. BMI was calculated as weight divided by height squared $\left(\mathrm{kg} / \mathrm{m}^{2}\right)$. Gender- and age-specific z-scores were also calculated (14). International age- and gender-specific cut-off points (14) were used to establish BMI-categories (underweight/normal weight/overweight/obese).

A set of skinfold thicknesses (biceps, triceps, subscapular, suprailiac, thigh) and circumferences (relaxed arm, flexed upper arm, waist, hip, upper thigh) were measured three consecutive times on the left side of the body with a skinfold calliper (Holtain, Crymych, UK) (the nearest $0.2 \mathrm{~mm}$ ), and with a non-elastic tape (Seca 200) to the nearest $0.1 \mathrm{~cm}$ respectively. Fat mass (FM) in $\mathrm{kg}$ and fat free mass (FFM) in kg were analysed by means of a tetrapolar technique (BIA 101 AKERN SRL, Pontassieve (FI), Italy). An index for these parameters was created as fat mass index and fat free mass index (FMI and FFMI, respectively) dividing the mass of each one by the square of the height $\left(\mathrm{kg} / \mathrm{m}^{2}\right)$. Physical examination was performed by a physician aiming to classify the adolescents into 1 of the 5 stages of pubertal maturity defined by Tanner and Whitehouse (15).

Physical activity. Physical activity was measured using accelerometers during a 7-d period (Actigraph GT1M, Manufacturing Technology Inc., Pensacola, FL). The time engaged per day at moderate PA was calculated based upon a blanket cut-off of 2,000 counts per minute (cpm), which is approximately equivalent to an intensity of a brisk walk $(4.5 \mathrm{~km} / \mathrm{h})$. The time engaged per day in vigorous PA was calculated based upon a blanket cutoff of 4,000 cpm (16). The cutoffs to define intensity categories were: moderate PA (2,000-3,999 cpm) and vigorous PA $(>4,000 \mathrm{cpm})$ (16). Sleep duration was assessed by means of a self-administered questionnaire. 
Physical fitness. The health-related physical fitness components assessed using the physical fitness tests from the Eurofit battery (17) included; cardiorespiratory physical fitness by $20-\mathrm{m}$ shuttle run test $(20 \mathrm{~m}$ sr) described by Léger et al. (18), upper-body muscular strength by handgrip test (maximum handgrip strength assessment by dynamometer), lower body muscular strength by standing broad jump (lower limb explosive strength assessment), speed-agility by the $4 \times 10-\mathrm{m}$ shuttle run test $(4 \times 10 \mathrm{sr})$ and flexibility by the sit and reach test in $\mathrm{cm}$. The scientific rationale for the selection of all of these tests as well as their reliability in young people was previously published. All the tests were performed twice; the best score was retained, except the 20-m sr which was performed only once.

Dietary assessment. A repeated 24-h recall was selected to be the most suitable method to get population means and distributions by the European Consumption Survey Method (EFCOSUM) project (19). Mean daily calcium and vitamin D intakes were estimated from two non-consecutive 24-h recalls using the HELENA-DIAT (Dietary Assessment Tool) software (20). A validation study by Vereecken et al. (20) indicated that HELENADIAT showed good agreement with an intervieweradministered 24-h recall for all nutrients, including calcium and vitamin D.

Dietary data were linked to the German Food Code and Nutrient Data Base [BLS (Bundeslebensmittelschlüssel) version II.3.1. 2005]. The Multiple Source Method (MSM) provided by the German Institute of Human Nutrition Potsdam-Rehbrücke (DIfE) as a web-based program package was used to account for within-person variation in the 24-h dietary recalls (21). With this method dietary data was corrected for between- and within-person variability. Only underreporters were excluded from all analyses. Underreporting was considered with the estimated basal metabolic rate $<0.96$ (22). Dietary intake data for Heraklion and Pecs were not available due to logistical reasons.

Supplementation. Information on vitamin supplementation was obtained via the clinical anamnesis of the adolescents [case report form (CRF)]. Adolescents were asked about taking any micronutrient supplement and were classified into two groups: supplement and non-supplement users.

Socioeconomic status (SES). The complete description of the self-reported socioeconomic questionnaire has been published elsewhere (23). Information on SES was determined by means of a self-administered questionnaire. The Family Affluence Scale (FAS) was calculated, based on a model developed by Currie et al. (24) but slightly adapted by replacing the item on holidays by Internet at home.

Specimen collection and biochemical analyses. Fasting blood samples were collected by venipuncture at school between eight and ten o'clock in the morning. For the measurement of vitamin D, blood was collected in EDTA tubes, immediately placed on ice, and centrifuged within $30 \mathrm{~min}$ (3,500 rpm for $15 \mathrm{~min})$. The supernatant fluid was transported at a stable temperature of $4-7^{\circ} \mathrm{C}$ to the central laboratory in Bonn, and stored there at $-80^{\circ} \mathrm{C}$ until assayed at Bonn University (25).

Vitamin D status. Plasma 25(OH)D was analysed by ELISA using a kit (OCTEIA 25-Hydroxy Vitamin D) from Immunodiagnostic System (Germany) and measured with a Sunrise ${ }^{\mathrm{TM}}$ Photometer by TECAN (Germany). The IDS OCTEIA 25-Hydroxy Vitamin D kit is an enzyme immunoassay intended for the quantitative determination of $25(\mathrm{OH}) \mathrm{D}$ and other hydroxylated metabolites in human serum or plasma. The sensitivity of this method is $5 \mathrm{nmol} / \mathrm{L} 25(\mathrm{OH}) \mathrm{D}$ and the variation is below $6 \%$. The mean recovery of $25(\mathrm{OH}) \mathrm{D}$ is $101 \%$. The CV for the method was below $1 \%$. Plasma $25(\mathrm{OH}) \mathrm{D}$ concentrations were classified in four groups for comparisons: (vitamin D sufficiency/optimal levels $\geq 75 \mathrm{nmol} / \mathrm{L}$; insufficiency 50-75 nmol/L; deficiency 27.5-49.99 nmol/L and severe deficiency $<27.5 \mathrm{nmol} / \mathrm{L}$ ), following international guidelines (26).

Retinol, $\alpha$-tocopherol and $\beta$-carotene status. Retinol, $\beta$-carotene, and $\alpha$-tocopherol were analysed by reversed phase high-performance liquid chromatography using UV detection (RP-HPLC) (Sykam Gilching, Germany) in serum. The vacutainer was centrifuged for $15 \mathrm{~min}$ at $3,500 \mathrm{rpm}$, at $4^{\circ} \mathrm{C}$. Standards $(\beta$-carotene, retinol, $\alpha$-tocopherol) hexane and isopropanol were obtained from Sigma Aldrich (Germany) and were all HPLCgrade. The variation of the method is below 3\% for all the vitamins. The samples were stable over $24 \mathrm{~h}$ at room temperature (coefficient of variation $\alpha$-tocopherol $=4.6 \%$; retinol vitamin $A=3.2 \%$ ).

VDR rs1544410 polymorphism. VDR genotype in its polymorphism (rs1544410) on chromosomes 7 and 12 blood for desoxyribonucleic acid (DNA) isolation was collected in EDTA tubes stored at IEL and sent to the Genomic Analysis Laboratory at the Institut Pasteur de Lille in France. DNA was extracted from white blood cells with the Puregene kit (QIAGEN, Courtaboeuf, France) and stored at $-80^{\circ} \mathrm{C}$. Samples were genotyped by an Illumina system, using the VeraCode technology. The genotyping success rate for the rs 1544410 was $>99 \%$.

Seasonality. The original variable "blood drawing date" was used to compute seasonality similarly to previous studies (27): Winter (1; January through March), Spring (2; April through June), and Autumn (3; October through December). Blood drawing was performed during the academic year.

Latitude. The latitude of each city was obtained from http://maps.google.es/. Latitudes of the involved cities were: Stockholm $\left(59^{\circ} 33^{\prime} \mathrm{N}\right)$, Athens $\left(37^{\circ} 98^{\prime}\right.$ $\mathrm{N})$, Heraklion $\left(35^{\circ} 33^{\prime} \mathrm{N}\right)$, Rome $\left(41^{\circ} 89^{\prime} \mathrm{N}\right)$, Zaragoza $\left(41^{\circ} 66^{\prime} \mathrm{N}\right)$, Pecs $\left(46^{\circ} 07^{\prime} \mathrm{N}\right)$, Ghent $\left(51^{\circ} 06^{\prime} \mathrm{N}\right)$, Lille $\left(50^{\circ} 63^{\prime} \mathrm{N}\right)$, Dortmund $\left(51^{\circ} 51^{\prime} \mathrm{N}\right)$, and Vienna $\left(48^{\circ} 21^{\prime}\right.$ $\mathrm{N})$. To make use of this, data latitudes were added to the database as numeric variables with two decimals (i.e. Stockholm $=59.55)$.

Statistical analysis. All the variables showed a normal distribution and residuals showed a satisfactory pattern. Descriptive values are shown as means \pm standard deviation unless otherwise stated. The independent samples ANOVA test was used to analyse differences in 
Table 1. Descriptive characteristics of the total study sample stratified by $25(\mathrm{OH}) \mathrm{D}$ concentrations $(\mathrm{nmol} / \mathrm{L})$ shown as mean \pm SD.

\begin{tabular}{|c|c|c|c|c|c|}
\hline & $\begin{array}{c}\text { All } \\
n=1,006\end{array}$ & $\begin{array}{c}25(\mathrm{OH}) \mathrm{D} \\
(<27.5 \mathrm{nmol} / \mathrm{L}) \\
n=79\end{array}$ & $\begin{array}{c}25(\mathrm{OH}) \mathrm{D} \\
(27.5-49.9 \mathrm{nmol} / \mathrm{L}) \\
n=299\end{array}$ & $\begin{array}{c}25(\mathrm{OH}) \mathrm{D} \\
(50-74.9 \mathrm{nmol} / \mathrm{L}) \\
n=422\end{array}$ & $\begin{array}{c}25(\mathrm{OH}) \mathrm{D} \\
(>75 \mathrm{nmol} / \mathrm{L}) \\
n=206\end{array}$ \\
\hline & Mean \pm SD & Mean \pm SD & Mean \pm SD & Mean \pm SD & Mean \pm SD \\
\hline Age & $14.7 \pm 11$ & $14.8 \pm 1.2$ & $14.5 \pm 1.1$ & $14.6 \pm 1.1$ & $14.9 \pm 1.1$ \\
\hline $\begin{array}{l}\text { Tanner stage } \\
\quad(\text { I, II, III, IV, V)\% }\end{array}$ & $1 / 5 / 19 / 44 / 31$ & $3 / 2 / 13 / 58 / 24$ & $0 / 6 / 20 / 43 / 31$ & $1 / 5 / 20 / 45 / 29$ & $1 / 4 / 18 / 39 / 38$ \\
\hline \multicolumn{6}{|l|}{ Body composition } \\
\hline Height (cm) & $165.4 \pm 9.3$ & $164.0 \pm 10.0$ & $165.3 \pm 9.6$ & $165.7 \pm 9.0$ & $165.5 \pm 9.3$ \\
\hline BMI z-score & $0.5 \pm 1.1$ & $0.4 \pm 1.0$ & $0.5 \pm 1.2$ & $0.5 \pm 1.1$ & $0.3 \pm 1.0$ \\
\hline Body fat $\%$ & $23.5 \pm 9.4$ & $23.2 \pm 9.3$ & $23.1 \pm 9.6$ & $24.1 \pm 9.4$ & $23.1 \pm 9.0$ \\
\hline FFM (kg) & $44.1 \pm 8.0$ & $43.2 \pm 7.8$ & $44.3 \pm 8.5$ & $44.3 \pm 7.9$ & $43.9 \pm 7.8$ \\
\hline FM index $\left(\mathrm{kg} / \mathrm{m}^{2}\right)$ & $5.3 \pm 3.0$ & $5.1 \pm 2.9$ & $5.2 \pm 3.1$ & $5.5 \pm 3.1$ & $5.0 \pm 2.6$ \\
\hline FFM index $\left(\mathrm{kg} / \mathrm{m}^{2}\right)$ & $16.8 \pm 1.8$ & $16.7 \pm 1.7$ & $16.9 \pm 1.9$ & $16.9 \pm 1.8$ & $16.5 \pm 1.5$ \\
\hline \multicolumn{6}{|l|}{ Biochemical markers } \\
\hline 25(OH)D (nmol/L) & $58.3 \pm 2.7$ & $22.0 \pm 4.6$ & $41.3 \pm 5.9$ & $61.4 \pm 6.9$ & $92.3 \pm 17.4$ \\
\hline Retinol (nmol/L) & $1,245.5 \pm 377.6$ & $1,210.8 \pm 355.9$ & $1,202.4 \pm 336.4$ & $1,245.8 \pm 373.1$ & $1,345.2 \pm 441.6$ \\
\hline$\alpha$-Tocopherol (nmol/L) & $4,257.0 \pm 903.0$ & $4,343.0 \pm 903.0$ & $4,257.0 \pm 903.0$ & $4,214.0 \pm 946.0$ & $4,343.0 \pm 860.0$ \\
\hline$\beta$-Carotene $(\mathrm{nmol} / \mathrm{L})$ & $455.2 \pm 310.4$ & $431.2 \pm 251.3$ & $414.0 \pm 253.7$ & $473.5 \pm 352.8$ & $490.7 \pm 312.3$ \\
\hline \multicolumn{6}{|l|}{ Genetic polymorphism } \\
\hline $\mathrm{SNP}(\mathrm{BB}, \mathrm{Bb}, \mathrm{bb}) \%$ & $16 / 48 / 36$ & $14 / 54 / 32$ & $15 / 51 / 34$ & $16 / 47 / 37$ & $18 / 44 / 38$ \\
\hline \multicolumn{6}{|l|}{ Dietary intake } \\
\hline Calcium intake (mg/d) & $855.7 \pm 515.7$ & $806.0 \pm 532.9$ & $845.0 \pm 461.1$ & $868.5 \pm 494.5$ & $864.2 \pm 592.6$ \\
\hline Vitamin D intake ( $\mu \mathrm{g} / \mathrm{d})$ & $2.1 \pm 1.0$ & $2.1 \pm 0.9$ & $2.0 \pm 0.8$ & $2.1 \pm 1.0$ & $2.2 \pm 1.2$ \\
\hline Supplements (yes/no) \% & 11/89 & $5 / 95$ & $9 / 91$ & $13 / 87$ & $13 / 87$ \\
\hline \multicolumn{6}{|l|}{ Activity } \\
\hline Sleep time $(\mathrm{h})$ & $8.1 \pm 1.2$ & $8.1 \pm 1.4$ & $8.2 \pm 1.3$ & $8.1 \pm 1.1$ & $8.0 \pm 1.1$ \\
\hline $\mathrm{PA}(\mathrm{min})$ & $59.2 \pm 24.5$ & $58.3 \pm 23.6$ & $59.4 \pm 26.8$ & $60.4 \pm 24.2$ & $56.9 \pm 22.0$ \\
\hline \multicolumn{6}{|l|}{ Physical fitness tests } \\
\hline Hand grip strength (kg) & $30.2 \pm 8.7$ & $29.9 \pm 9.9$ & $30.2 \pm 8.9$ & $30.5 \pm 8.3$ & $29.9 \pm 8.7$ \\
\hline SBJump (cm) & $161.8 \pm 34.5$ & $160.8 \pm 38.2$ & $160.9 \pm 35.2$ & $161.6 \pm 32.1$ & $163.7 \pm 37.2$ \\
\hline $20 \mathrm{~m} \mathrm{sr}$ (stage) & $4.8 \pm 2.8$ & $4.9 \pm 3.0$ & $4.6 \pm 2.7$ & $4.8 \pm 2.7$ & $5.1 \pm 2.9$ \\
\hline $4 \times 10 \mathrm{sr}(\mathrm{s})$ & $12.2 \pm 1.3$ & $12.4 \pm 1.6$ & $12.2 \pm 1.3$ & $12.2 \pm 1.2$ & $12.2 \pm 1.3$ \\
\hline Flexibility $(\mathrm{cm})$ & $22.4 \pm 8.1$ & $21.3 \pm 7.7$ & $21.7 \pm 7.6$ & $22.9 \pm 8.3$ & $22.7 \pm 8.4$ \\
\hline \multicolumn{6}{|l|}{ Socioeconomic status } \\
\hline FAS & $4.5 \pm 1.8$ & $4.3 \pm 1.9$ & $4.3 \pm 1.9$ & $4.4 \pm 1.8$ & $4.9 \pm 1.8$ \\
\hline
\end{tabular}

Normally distributed variables are shown as mean \pm SD.

Four 25(OH)D groups by concentrations: 25(OH)D severe deficiency $(<27.5 \mathrm{nmol} / \mathrm{L}), 25(\mathrm{OH}) \mathrm{D}$ deficiency $(<50 \mathrm{nmol} / \mathrm{L})$, 25(OH)D insufficiency (50-74.9 nmol/L), 25(OH)D sufficiency ( $\geq 75 \mathrm{nmol} / \mathrm{L})$.

BMI: body mass index $\left(\mathrm{kg} / \mathrm{m}^{2}\right)$, FFM: fat free mass $(\mathrm{kg})$, FM index: fat mass index $\left(\mathrm{kg} / \mathrm{m}^{2}\right), \mathrm{FFM}$ index: fat free mass index $(\mathrm{kg} /$ $\mathrm{m}^{2}$ ), PA: physical activity unless at moderate intensity (min), SBJump: standing broad jump (cm), $20 \mathrm{~m}$ sr: $20 \mathrm{~m}$ shuttle run, $4 \times 10$ sr: $4 \times 10 \mathrm{~m}$ shuttle run (s), FAS: family affluence scale.

Genetic: SNP on chromosone $7-1 ; \mathrm{B}=$ wild type allele/b=mutant type allele.

25(OH)D between concentration groups. A partial Pearson's correlation coefficient stratified by sex was used to assess the association between $25(\mathrm{OH}) \mathrm{D}$ concentrations and independent predictors controlled for age and centre.

A stepwise multivariate linear regression model stratified by sex examined the independent associations between 25(OH)D concentrations and age (28), Tanner stage, season, latitude $\left({ }^{\circ}\right)$, BMI z-score, fat mass (\%), FFM $(\mathrm{kg})$, FMI $\left(\mathrm{kg} / \mathrm{m}^{2}\right)$, FFMI $\left(\mathrm{kg} / \mathrm{m}^{2}\right)$, genetic polymorphism (rs1544410), retinol (nmol/L), $\alpha$-tocopherol (nmol/L), $\beta$-carotene $(\mathrm{nmol} / \mathrm{L})$, calcium intake $(\mathrm{mg} / \mathrm{d})$, vitamin $\mathrm{D}$ intake $(\mu \mathrm{g} / \mathrm{d})$, micronutrient supplementation, PA (cpm), fitness and socioeconomic status (Table 3).

All of the analyses were performed using the Statistical Package for Social Sciences software (SPSS, version 15.0 for Windows; SPSS, Chicago, IL), and values of $p<0.05$ were considered statistically significant. Figures were created using Sigmaplot (version 10.0 for Windows; Systat Software, San José, CA).

\section{RESULTS}

Table 1 shows descriptive characteristics of the sample stratified by $25(\mathrm{OH}) \mathrm{D}$ concentration. Pearson partial correlation coefficients by sex adjusted by age and centre are shown in Table 2. In males, season (sunny 
Table 2. Partial Pearson's coefficient correlations between the 25(OH)D concentration and body composition, dietary intake, muscular strength, cardiovascular fitness and socioeconomic status adjusted for age, sex and centre (latitude).

\begin{tabular}{|c|c|c|c|c|}
\hline & Males & $p$-value & Females & $p$-value \\
\hline $\begin{array}{l}\text { Season as date of blood draw } \\
\text { (sunny months) }\end{array}$ & $0.284^{* *}$ & 0.001 & $0.343^{* *}$ & 0.001 \\
\hline \multicolumn{5}{|l|}{ Body composition } \\
\hline Height $(\mathrm{cm})$ & -0.026 & 0.727 & 0.108 & 0.223 \\
\hline BMI z-score & $-0.168^{*}$ & 0.048 & -0.045 & 0.77 \\
\hline Body fat $\%$ & -0.141 & 0.094 & 0.027 & 0.51 \\
\hline FFM $(\mathrm{kg})$ & -0.090 & 0.464 & -0.002 & 0.471 \\
\hline FM index $\left(\mathrm{kg} / \mathrm{m}^{2}\right)$ & -0.141 & 0.095 & -0.008 & 0.628 \\
\hline FFM index $\left(\mathrm{kg} / \mathrm{m}^{2}\right)$ & -0.121 & 0.247 & 0.030 & 0.295 \\
\hline \multicolumn{5}{|l|}{ Biochemical markers } \\
\hline Retinol (nmol/L) & $0.187^{*}$ & 0.033 & 0.132 & 0.112 \\
\hline$\beta$-Carotene $(\mathrm{nmol} / \mathrm{L})$ & 0.144 & 0.112 & 0.041 & 0.989 \\
\hline$\alpha$-Tocopherol $(\mathrm{nmol} / \mathrm{L})$ & 0.053 & 0.564 & -0.003 & 0.767 \\
\hline \multicolumn{5}{|l|}{ Dietary intake } \\
\hline Calcium intake $(\mathrm{mg} / \mathrm{d})$ & -0.006 & 0.444 & 0.168 & 0.063 \\
\hline Vitamin D intake $(\mu \mathrm{g} / \mathrm{d})$ & -0.065 & 0.778 & -0.005 & 0.783 \\
\hline Supplement intake & 0.109 & 0.207 & $0.204^{*}$ & 0.025 \\
\hline \multicolumn{5}{|l|}{ Activity } \\
\hline Sleep (h) & -0.019 & 0.908 & $-0.243^{*}$ & 0.01 \\
\hline MVPA (min) & 0.008 & 0.933 & 0.05 & 0.957 \\
\hline \multicolumn{5}{|l|}{ Physical fitness tests } \\
\hline Handgrip strength (kg) & -0.107 & 0.188 & 0.068 & 0.481 \\
\hline SBJump $(\mathrm{cm})$ & 0.051 & 0.745 & 0.068 & 0.639 \\
\hline $20 \mathrm{~m}$ sr (stage) & 0.144 & 0.057 & 0.110 & 0.385 \\
\hline $4 \times 10$ sr $(\mathrm{s})$ & -0.066 & 0.484 & -0.118 & 0.148 \\
\hline Flexibility $(\mathrm{cm})$ & 0.019 & 0.936 & $0.265^{*}$ & 0.002 \\
\hline \multicolumn{5}{|l|}{ Socioeconomic status } \\
\hline FAS & 0.173 & 0.055 & 0.089 & 0.343 \\
\hline
\end{tabular}

** Correlation is significant at the 0.01 level (2-tailed).

* Correlation is significant at the 0.05 level (2-tailed).

BMI: body mass index $\left(\mathrm{kg} / \mathrm{m}^{2}\right)$, FFM: fat free mass $(\mathrm{kg})$, FM index: fat mass index $\left(\mathrm{kg} / \mathrm{m}^{2}\right)$, FFM index: fat free mass index $(\mathrm{kg} /$ $\mathrm{m}^{2}$ ), MVPA: physical activity unless at moderate intensity ( $\left.\mathrm{min}\right)$, SBJump: standing broad jump (cm), $20 \mathrm{~m}$ sr: $20 \mathrm{~m}$ shuttle run, $4 \times 10$ sr: $4 \times 10 \mathrm{~m}$ shuttle run (s), FAS: family affluence scale.

months) assessed by date of blood draw $(r=0.284$, $p<0.01)$, BMI $(r=-0.168, p<0.05)$ and retinol concentration $(r=0.187, p<0.05)$ significantly correlated with $25(\mathrm{OH}) \mathrm{D}$ concentrations. In females, season $(r=0.343$, $p<0.01)$, supplement intake $(r=0.204, p<0.05)$, sleep time in hours $(r=-0.243, p<0.05)$ and flexibility $(r=0.265, p<0.05)$ were significantly correlated with $25(\mathrm{OH}) \mathrm{D}$ concentrations.

Table 3 presents the results of the stepwise linear regression split by sex. In males, linear regression of $25(\mathrm{OH}) \mathrm{D}$, suggested that $(1)$ winter season $(\beta=-0.364$; $p<0.01)$, (2) higher latitudes $(\beta=-0.246$; $p<0.01)$ (3) BMI z-score $(\beta=-0.198 ; p<0.05)$ and (4) retinol concentration $(\beta=0.171 ; p<0.05)$ independently influenced 25(OH)D concentrations. In females, (1) winter season $(\beta=-0.370 ; p<0.01)$ (2) sleep time $(\beta=-0.231 ; p<0.01) \quad(3)$ supplements $(\beta=0.221$; $p<0.05$ ) (4) flexibility $(\beta=0.184 ; p<0.05)$ (5) body fat $\% \quad(\beta=0.201 ; p<0.05)$ (6) BMI z-score $(\beta=-0.272$; $p<0.05)$ (7) higher latitudes $(\beta=-0.219 ; p<0.01)$ and (8) handgrip strength $(\beta=0.206 ; p<0.05)$ independently influenced $25(\mathrm{OH}) \mathrm{D}$ concentrations.

Figure 1 presents differences in $25(\mathrm{OH}) \mathrm{D}$ concen- trations by season. The highest levels were observed in autumn $(66.3+25.0 \mathrm{nmol} / \mathrm{L})$, followed by spring $(63.9+25.0 \mathrm{nmol} / \mathrm{L})$, and both presented significant differences with winter levels $(46.9+18.0 \mathrm{nmol} / \mathrm{L})$ (both $p<0.001)$.

Figure 2 presents the differences in $25(\mathrm{OH}) \mathrm{D}$ concentrations according by centre (latitude). The highest levels were observed in Southern countries of Europe such as Rome $(70.0+20.0 \mathrm{nmol} / \mathrm{L})$, Athens $(68.2+20.7$ $\mathrm{nmol} / \mathrm{L})$, Zaragoza $(62.9+19.2 \mathrm{nmol} / \mathrm{L})$ and also Vienna $(63.7+31.7 \mathrm{nmol} / \mathrm{L})$. Significantly lower levels were found in Dortmund $(49.3+21.8 \mathrm{nmol} / \mathrm{L})$, Heraklion $(51.3+13.4 \mathrm{nmol} / \mathrm{L})$ and Ghent $(52.3+22.8 \mathrm{nmol} / \mathrm{L})$ $(p<0.01)$ compared to the Northern countries. None of the cities had a mean level above the proposed cut-off for sufficiency of $75 \mathrm{nmol} / \mathrm{L}$.

\section{DISCUSSION}

Despite the numerous scientific studies performed in the last years in relation to vitamin D status and its effect on various health indicator outcomes $(29,30)$, limited comparable data on vitamin D status and its associated factors in European adolescents exist. To the best 
Table 3. Stepwise multiple regression model for 25(OH)D $(\mathrm{nmol} / \mathrm{L})$ stratified by gender.

\begin{tabular}{|c|c|c|c|c|c|c|c|c|c|}
\hline \multicolumn{10}{|c|}{$25(\mathrm{OH}) \mathrm{D}$ concentrations $(\mathrm{nmol} / \mathrm{L})$} \\
\hline \multicolumn{5}{|c|}{ Males } & \multicolumn{5}{|c|}{ Females } \\
\hline & $\beta$ & $\begin{array}{c}\text { Partial } \\
\text { corr. }\end{array}$ & $p$-value & $R^{2}$ & & $\beta$ & $\begin{array}{c}\text { Partial } \\
\text { corr. }\end{array}$ & $p$-value & $R^{2}$ \\
\hline Model 1 & & & & 0.136 & Model 1 & & & & 0.129 \\
\hline Season winter & -0.378 & -0.378 & $<0.001$ & & Season winter & -0.370 & -0.370 & $<0.001$ & \\
\hline Model 2 & & & & 0.190 & Model 2 & & & & 0.175 \\
\hline Season winter & -0.364 & -0.377 & $<0.001$ & & Season winter & -0.359 & -0.371 & $<0.001$ & \\
\hline Higher latitudes & -0.246 & -0.265 & 0.003 & & Sleep $(h)$ & -0.231 & -0.248 & 0.011 & \\
\hline Model 3 & & & & 0.221 & Model 3 & & & & 0.217 \\
\hline Season winter & -0.387 & -0.403 & $<0.001$ & & Season winter & -0.366 & -0.387 & $<0.001$ & \\
\hline Higher latitudes & -0.283 & -0.303 & 0.001 & & Sleep $(h)$ & -0.229 & -0.254 & 0.009 & \\
\hline BMI z-score & -0.198 & -0.216 & 0.017 & & Supplements & 0.221 & 0.245 & 0.012 & \\
\hline Model 4 & & & & 0.244 & Model 4 & & & & 0.243 \\
\hline Season winter & -0.376 & -0.399 & $<0.001$ & & Season winter & -0.348 & -0.375 & $<0.001$ & \\
\hline Higher latitudes & -0.275 & -0.300 & 0.001 & & Sleep (h) & -0.232 & -0.262 & 0.007 & \\
\hline BMI z-score & -0.224 & -0.244 & 0.007 & & Supplements & 0.199 & 0.226 & 0.022 & \\
\hline \multirow{31}{*}{ Retinol (nmol/L) } & 0.171 & 0.192 & 0.035 & & Flexibility & 0.184 & 0.208 & 0.035 & \\
\hline & & & & & Model 5 & & & & 0.275 \\
\hline & & & & & Season winter & -0.338 & -0.374 & $<0.001$ & \\
\hline & & & & & Sleep $(h)$ & -0.227 & -0.263 & 0.007 & \\
\hline & & & & & Supplements & 0.195 & 0.227 & 0.022 & \\
\hline & & & & & Flexibility & 0.239 & 0.264 & 0.007 & \\
\hline & & & & & Body fat $\%$ & 0.201 & 0.226 & 0.022 & \\
\hline & & & & & Model 6 & & & & 0.296 \\
\hline & & & & & Season winter & -0.339 & -0.382 & $<0.001$ & \\
\hline & & & & & Sleep (h) & -0.227 & -0.268 & 0.007 & \\
\hline & & & & & Supplements & 0.206 & 0.243 & 0.014 & \\
\hline & & & & & Flexibility & 0.253 & 0.283 & 0.004 & \\
\hline & & & & & Body fat $\%$ & 0.420 & 0.291 & 0.003 & \\
\hline & & & & & BMI z-score & -0.272 & -0.198 & 0.047 & \\
\hline & & & & & Model 7 & & & & 0.331 \\
\hline & & & & & Season winter & -0.301 & -0.348 & $<0.001$ & \\
\hline & & & & & Sleep (h) & -0.194 & -0.235 & 0.018 & \\
\hline & & & & & Supplements & 0.230 & 0.275 & 0.006 & \\
\hline & & & & & Flexibility & 0.232 & 0.267 & 0.007 & \\
\hline & & & & & Body fat $\%$ & 0.497 & 0.340 & 0.001 & \\
\hline & & & & & BMI z-score & -0.386 & -0.269 & 0.007 & \\
\hline & & & & & Higher latitudes & -0.219 & -0.243 & 0.015 & \\
\hline & & & & & Model 8 & & & & 0.359 \\
\hline & & & & & Season winter & -0.270 & -0.320 & 0.001 & \\
\hline & & & & & Sleep (h) & -0.198 & -0.246 & 0.014 & \\
\hline & & & & & Supplements & 0.242 & 0.295 & 0.003 & \\
\hline & & & & & Flexibility & 0.208 & 0.244 & 0.015 & \\
\hline & & & & & Body fat $\%$ & 0.580 & 0.387 & 0.000 & \\
\hline & & & & & BMI z-score & -0.530 & -0.337 & 0.001 & \\
\hline & & & & & Higher latitudes & -0.292 & -0.306 & 0.002 & \\
\hline & & & & & Handgrip strength & 0.206 & 0.227 & 0.024 & \\
\hline
\end{tabular}

All variables were entered together into a stepwise regression model. BMI: body mass index $\left(\mathrm{kg} / \mathrm{m}^{2}\right)$.

of the authors' knowledge, this is the first study aiming to examine the association between a large number of individual, environmental and genetic determinants and vitamin D status in European adolescents.

Our findings suggest that season, latitude, adiposity, fitness, sleep time and micronutrient supplementation explained most of the variance observed in $25(\mathrm{OH}) \mathrm{D}$ concentrations in this sample of adolescents. Our results are in agreement with other studies conducted in international settings (31). For instance, Dong et al. (8) in the United States suggested that there are positive associations between vitamin D concentrations and healthy lifestyle factors including PA, adiposity and cardiovascular fitness, being independent of gender, sexual matura- 


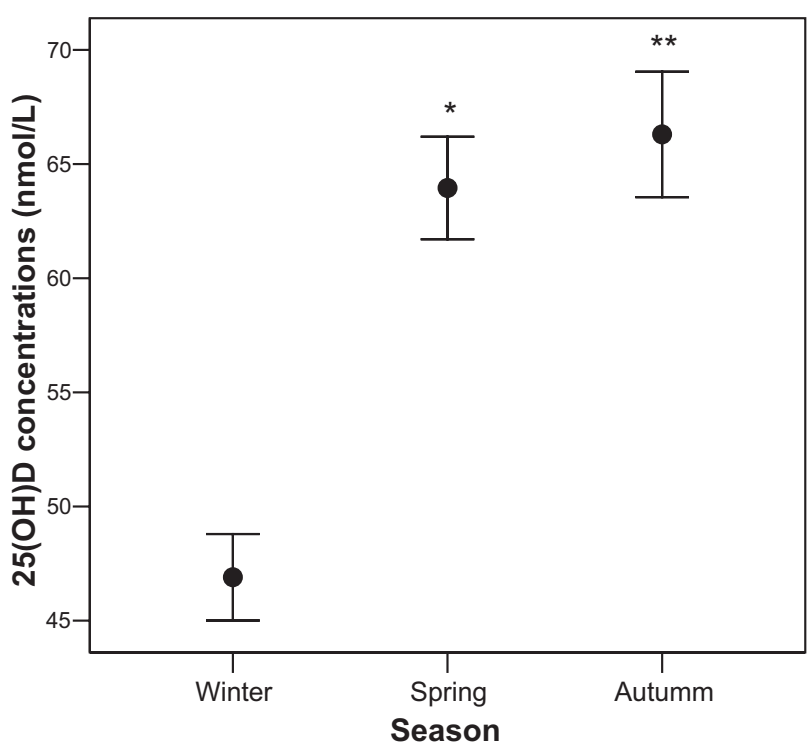

Fig. 1. Mean 25(OH)D concentrations (nmol/L) by seasons during the academic year (autumn, winter and spring). ${ }^{*} p<0.001$ significant differences between winter and spring for $25(\mathrm{OH}) \mathrm{D}$ concentrations. ${ }^{* *} p<0.001$ significant differences between winter and autumn for $25(\mathrm{OH}) \mathrm{D}$ concentrations.

tion and height. Similarly, Bener et al. (32) concluded that diet, lack of exposure to sunlight, outdoor activities and PA were the main associated factors of vitamin D deficiency in a young Qatari population. Arguelles et al. (4) suggested that gender, summer season, and high PA significantly increased 25(OH)D levels in a Chinese population.

Seasonal differences explained most of the variance of the $25(\mathrm{OH}) \mathrm{D}$ concentrations, confirming the results of other studies across Europe $(6,7,33,34)$. Casual exposure to sunlight is thought to provide most of the vitamin D requirements of the human population (6). Our results have suggested that the highest values of $25(\mathrm{OH}) \mathrm{D}$ concentrations were obtained in autumn following exposure to the summer period. On the other hand, the winter season was negatively related with $25(\mathrm{OH}) \mathrm{D}$ concentrations. Highest 25(OH)D concentrations were obtained in the Southern cities of Europe and the lowest in the Northern cities, which confirms results of other studies $(6,33,34)$. Unexpectedly, we observed low mean concentrations in Heraklion, which could be attributed to seasonal influences in blood extraction; i.e. due to local logistics, blood sampling was performed only in February and March, and was not distributed throughout the academic year. This indicates that the date of blood extraction at each latitude influences 25(OH)D concentrations (3). Nevertheless, the results are of interest, due to the low concentrations obtained in Heraklion, which could indicate a risk during the winter months even in the Mediterranean countries. These findings are not surprising and confirm others indicating higher concentrations during sunny months $(35,36)$. However, seasonality should be considered together with latitude as UVB radiation of the appro-

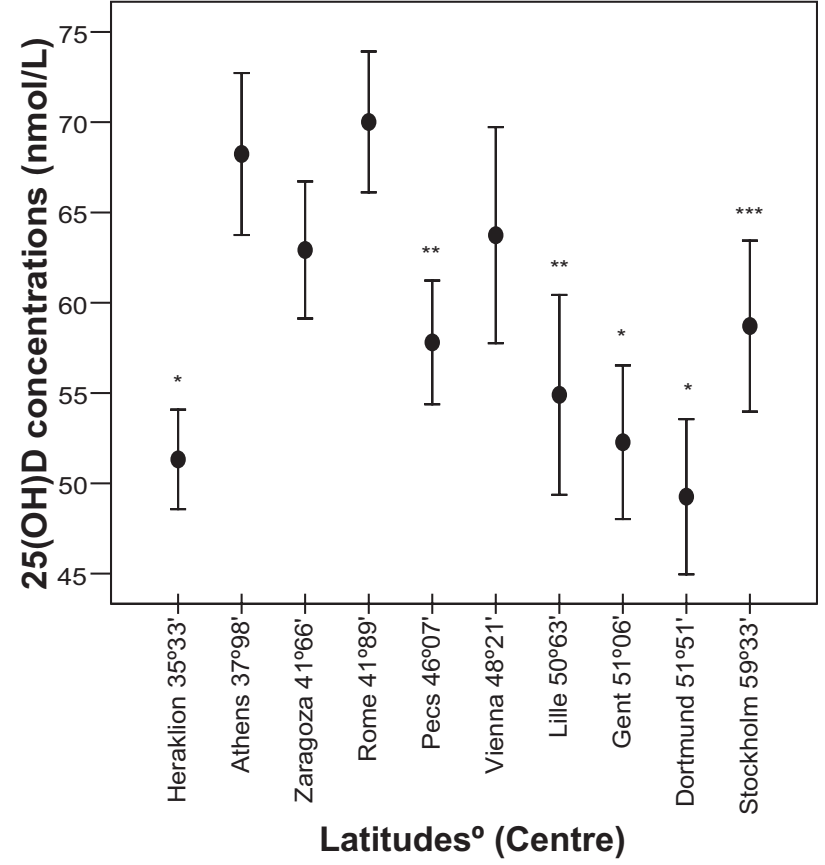

Fig. 2. Mean 25(OH)D concentrations (nmol/L) by centres at different latitudes. ${ }^{*} p<0.01$ significant differences between centres with highest $25(\mathrm{OH}) \mathrm{D}$ concentrations (Rome, Athens, Vienna and Zaragoza) and centres with lowest 25(OH)D concentrations. ${ }^{* *} p<0.01$ significant differences between Lille and Pecs respect to Athens and Rome for $25(\mathrm{OH}) \mathrm{D}$ concentrations. *** $p<0.01$ significant differences between Stockholm respect to Rome 25(OH)D concentrations.

priate wavelength, especially during winter time (37), might be absent in the latitudes of the hemisphere not close to the Ecuador. Moreover, the comparison with other studies in Europe are difficult because of the lack of standardization (7).

Current research has observed an inverse relation between vitamin D and BMI (38). Our results have similarly indicated a negative influence of increasing BMI with decreasing 25(OH)D concentrations in both sexes. Enhanced sequestration of vitamin D in fat could possibly explain the observed relation (39). In the study by Dong et al. vitamin D status was also influenced by adiposity (8). On the other hand, others failed to identify an association in paediatric populations $(40,41)$.

To the authors' knowledge, this is also the first study to examine the relation between a genetic polymorphism (rs1544410) and 25(OH)D concentrations in European adolescents. Our results did not show a significant contribution of polymorphism rs1544410 on vitamin D levels in European adolescents. Previous European studies in adults using different methods of genetic analyses have estimated around a $30-45 \%$ heritability in vitamin D status and demonstrated that nutrient levels are fractionally under some genetic control. On the other hand, a study of Chinese adolescents twins suggested a strong genetic influence on 25(OH)D level in males (4).

Other possible determinants like diet, PA, physical fitness and SES should be taken into consideration when 
examining association of vitamin D status in adolescent populations. In our study, physical fitness, mainly for muscular strength and flexibility, and not PA highly explained 25(OH)D variability, showing a positive influence; these findings are in agreement with other studies $(42,43)$. Vitamin D is indeed the other steroid hormone that is important for muscle function and strength (43). Unlike our findings, Dong et al. (8) reported significantly positive associations between $25(\mathrm{OH}) \mathrm{D}$ levels and physical fitness in American adolescents. Examination of sleep duration, often associated with higher adiposity markers particularly in female adolescents (44), with $25(\mathrm{OH}) \mathrm{D}$ levels suggested negative relations in our female sample. It is possible that lack of sun exposure and outdoor activities while sleeping could be responsible for such observations but further analyses are needed.

Diet and micronutrient supplementation appeared as strong and positive determinants of vitamin D status and could be linked with the positive influence found for retinol levels on $25(\mathrm{OH}) \mathrm{D}$ concentrations. Supporting our positive results. Guillemant et al. (35) and LehtonenVeromaa et al. (34) observed a significant effect of supplementation on 25(OH)D serum levels in French and Finnish children $(34,45)$. This is in accordance with emerging evidence emphasizing the need of vitamin D supplementation in high-risk groups especially during winter months (46). There is, however, an ongoing debate on the influence of vitamin D supplementation or fortified foods in children and adolescents (46).

Abnormal calcium metabolism has been associated with weight gain (47), and high micronutrient intake is believed to prevent obesity, linked at the same time with vitamin D deficiency (48). The authors failed to identify any association with vitamin $\mathrm{D}$ intakes in agreement with Absoud et al. (31). On the contrary Bener et al. indicated low vitamin D intakes as the main determinant of vitamin D deficiency in young Qatari children (32).

In addition to the above limitations of season and latitude, dietary intake data for Heraklion and Pecs were not available which makes it more difficult to explain the observed low 25(OH)D levels found in these countries. Nevertheless, the HELENA study has several strengths. The sampling procedure and the strict standardization of the field work among the countries involved in the study avoided to a great extent the kind of confounding bias due to inconsistent protocols and different laboratory methods which makes comparing results from isolated studies difficult. The main contribution of our study is the use of a large pool of determinants, which for the first time provide a deeper understanding of vitamin D status in European adolescents. The majority of the studies only assess PA and BMI but not fitness nor FFM and FM in relation to vitamin D, which is an additional strength of our work.

In conclusion season, latitude, physical fitness, adiposity, sleep time and micronutrient supplementation strongly influence 25(OH)D concentrations in European adolescents. Controversial results found in our study like the influence of body composition and dietary intake, indicate the need for further research. In the absence of reference values and specific cut-off points for adolescents, determinant parameters which influence vitamin D concentrations should be also taken into account for further research.

\section{Disclosure}

The content of this paper reflects only the authors' view and the rest of HELENA study members are not responsible for it. The writing group takes sole responsibility for the content of this article.

\section{Acknowledgments}

The HELENA Study has taken place with the financial support of the European Community Sixth RTD Framework Programme (Contract FOOD-CT-2005-007034). The content of this article reflects only the authors' views and the European Community is not liable for any use that may be made of the information contained therein. Additional support came from the Spanish Ministry of Education (AGL2007-29784-E/ALI; AP-20053827, AP-2008-03806). Jara Valtueña was financially supported by the Universidad Politécnica de Madrid (CH/018/2008). Isabelle Sioen is financially supported by the Research Foundation-Flanders (Grant $\mathrm{n}^{\circ}$ : 1.2.683.11.N.00). Many thanks to to Adelheid Schuch for her contribution to laboratory work and to Laura Barrios for statistical assistance.

None of the authors had any conflict of interests.

Contributions of each author: MGG, LAM, MK, FG, JD, AK, YM, MF, KW and PS designed the HELENA study and conducted research (recruitment of adolescents, data collection, and blood sampling); JV, CB, and EA conducted research and performed blood analysis; JV, MGG, and IH performed the statistical analysis; JV, MGG and LAM wrote the paper. JV, IH, and JD participated in the data interpretation. JV, SG, MCG and IS participated in the literature search. JV, MGG and LAM had primary responsibility for final content. All authors read and approved the final manuscript.

\section{*HELENA Study Group}

Co-ordinator: Luis A. Moreno.

Core Group members: Luis A. Moreno, Fréderic Gottrand, Stefaan De Henauw, Marcela González-Gross, Chantal Gilbert.

Steering Committee: Anthony Kafatos (President), Luis A. Moreno, Christian Libersa, Stefaan De Henauw, Jackie Sáchez, Fréderic Gottrand, Mathilde Kesting, Michael Sjostrom, Dénes Molnár, Marcela GonzálezGross, Jean Dallongeville, Chantal Gilbert, Gunnar Hall, Lea Maes, Luca Scalfi.

Project Manager: Pilar Meléndez.

1. Universidad de Zaragoza (Spain): Luis A. Moreno, Jesús Fleta, José A. Casajús, Gerardo Rodríguez, Concepción Tomás, María I. Mesana, Germán Vicente-Rodríguez, Adoración Villarroya, Carlos M. Gil, Ignacio Ara, Juan Revenga, Carmen Lachen, Juan Fernández Alvira, 
Gloria Bueno, Aurora Lázaro, Olga Bueno, Juan F. León, Jesús Ma Garagorri, Manuel Bueno, Juan Pablo Rey López, Iris Iglesia, Paula Velasco, Silvia Bel, Theodora Mouratidou.

2. Consejo Superior de Investigaciones Científicas (Spain): Ascensión Marcos, Julia Wärnberg, Esther Nova, Sonia Gómez, Esperanza Ligia Díaz, Javier Romeo, Ana Veses, Mari Angeles Puertollano, Belén Zapatera, Tamara Pozo.

3. Université de Lille 2 (France): Laurent Beghin, Christian Libersa, Frédéric Gottrand, Catalina Iliescu, Juliana Von Berlepsch.

4. Research Institute of Child Nutrition Dortmund, Rheinische Friedrich-Wilhelms-Universität Bonn (Germany): Mathilde Kersting, Wolfgang Sichert-Hellert, Ellen Koeppen.

5. Pécsi Tudományegyetem (University of Pécs) (Hungary): Dénes Molnar, Eva Erhardt, Katalin Csernus, Katalin Török, Szilvia Bokor, Angster, Enikö Nagy, Orsolya Kovács, Judit Repásy.

6. University of Crete School of Medicine (Greece): Anthony Kafatos, Caroline Codrington, María Plada, Angeliki Papadaki, Katerina Sarri, Anna Viskadourou, Christos Hatzis, Michael Kiriakakis, George Tsibinos, Constantine Vardavas, Manolis Sbokos, Eva Protoyeraki, Maria Fasoulaki.

7. Institut für Ernährungs- und Lebensmittelwissenschaften-Ernährungphysiologie, Rheinische Friedrich Wilhelms Universität (Germany): Peter Stehle, Klaus Pietrzik, Marcela González-Gross, Christina Breidenassel, Andre Spinneker, Jasmin Al-Tahan, Miriam Segoviano, Anke Berchtold, Christine Bierschbach, Erika Blatzheim, Adelheid Schuch, Petra Pickert.

8. University of Granada (Spain): Manuel J. Castillo, Ángel Gutiérrez, Francisco B. Ortega, Jonatan R. Ruiz, Enrique G. Artero, Vanesa España-Romero, David Jiménez-Pavón, Palma Chillón.

9. Istituto Nazionale di Ricerca per gli Alimenti e la Nutrizione (Italy): Davide Arcella, Elena Azzini, Emma Barrison, Noemi Bevilacqua, Pasquale Buonocore, Giovina Catasta, Laura Censi, Donatella Ciarapica, Paola D’Acapito, Marika Ferrari, Myriam Galfo, Cinzia Le Donne, Catherine Leclercq, Giuseppe Maiani, Beatrice Mauro, Lorenza Mistura, Antonella Pasquali, Raffaela Piccinelli, Angela Polito, Raffaella Spada, Stefania Sette, Maria Zaccaria.

10. University of Napoli "Federico II" Department of Food Science (Italy): Luca Scalfi, Paola Vitaglione, Concetta Montagnese.

11. Ghent University (Belgium): Ilse De Bourdeaudhuij, Stefaan De Henauw, Tineke De Vriendt, Lea Maes, Christophe Matthys, Carine Vereecken, Mieke de Maeyer, Charlene Ottevaere.

12. Medical University of Vienna (Austria): Kurt Widhalm, Katharina Phillipp, Sabine Dietrich.

13. Harokopio University (Greece): Yannis Manios, Eva Grammatikaki, Zoi Bouloubasi, Tina Louisa Cook, Sofia Eleutheriou, Orsalia Consta, George Moschonis, Ioanna Katsaroli, George Kraniou, Stalo Papoutsou, Despoina Keke, Ioanna Petraki, Elena Bellou, Sofia Tana- gra, Kostalenia Kallianoti, Dionysia Argyropoulou, Katerina Kondaki, Stamatoula Tsikrika, Christos Karaiskos.

14. Institut Pasteur de Lille (France): Jean Dallongeville, Aline Meirhaeghe.

15. Karolinska Institutet (Sweden): Michael Sjöstrom, Patrick Bergman, María Hagströmer, Lena Hallström, Mårten Hallberg, Eric Poortvliet, Julia Wärnberg, Nico Rizzo, Linda Beckman, Anita Hurtig Wennlöf, Emma Patterson, Lydia Kwak, Lars Cernerud, Per Tillgren, Stefaan Sörensen.

16. Asociación de Investigación de la Industria Agroalimentaria (Spain): Jackie Sánchez-Molero, Elena Picó, Maite Navarro, Blanca Viadel, José Enrique Carreres, Gema Merino, Rosa Sanjuán, María Lorente, María José Sánchez, Sara Castelló.

17. Campden \& Chorleywood Food Research Association (United Kingdom): Chantal Gilbert, Sarah Thomas, Elaine Allchurch, Peter Burguess.

18. SIK-Institutet foer Livsmedel och Bioteknik (Sweden): Gunnar Hall, Annika Astrom, Anna Sverkén, Agneta Broberg.

19. Meurice Recherche \& Development asbl (Belgium): Annick Masson, Claire Lehoux, Pascal Brabant, Philippe Pate, Laurence Fontaine.

20. Campden \& Chorleywood Food Development Institute (Hungary): Andras Sebok, Tunde Kuti, Adrienn Hegyi.

21. Productos Aditivos SA (Spain): Cristina Maldonado, Ana Llorente.

22. Cárnicas Serrano SL (Spain): Emilio García.

23. Cederroth International AB (Sweden): Holger von Fircks, Marianne Lilja Hallberg, Maria Messerer.

24. Lantmännen Food RED (Sweden): Mats Larsson, Helena Fredriksson, Viola Adamsson, Ingmar Börjesson.

25. European Food Information Council (Belgium): Laura Fernández, Laura Smillie, Josephine Wills.

26. Universidad Politécnica de Madrid (Spain): Marcela González-Gross, Jara Valtueña, David JiménezPavón, Ulrike Albers, Raquel Pedrero, Agustín Meléndez, Pedro J. Benito, Juan José Gómez Lorente, David Cañada, Alejandro Urzanqui, Juan Carlos Ortiz, Francisco Fuentes, Rosa María Torres, Paloma Navarro.

\section{REFERENCES}

1) Foo LH, Zhang Q, Zhu K, Ma G, Trube A, Greenfield H, Fraser DR. 2009. Relationship between vitamin D status, body composition and physical exercise of adolescent girls in Beijing. Osteoporos Int 20: 417-425.

2) Gracia-Marco L, Valtueña J, Ortega FB, Perez-Lopez FR, Vicente-Rodriguez G, Breidenassel C, Ferrari M, Molnar D, Widhalm K, de Henauw S, Kafatos A, Diaz LE, Gottrand F, Maiani G, Stehle P, Castillo MJ, Moreno LA, Gonzalez-Gross M. 2012. Iron and vitamin status biomarkers and its association with physical fitness in adolescents: the HELENA study. J Appl Physiol 113: 566-573.

3) Gonzalez-Gross M, Valtueña J, Breidenassel C, Moreno LA, Ferrari M, Kersting M, De Henauw S, Gottrand F, Azzini E, Widhalm K, Kafatos A, Manios Y, Stehle P. 2012. Vitamin D status among adolescents in Europe: 
the Healthy Lifestyle in Europe by Nutrition in Adolescence study. Br J Nutr 107: 755-764.

4) Arguelles LM, Langman CB, Ariza AJ, Ali FN, Dilley K, Price H, Liu X, Zhang S, Hong X, Wang B, Xing H, Li Z, Zhang W, Xu X, Wang X. 2009. Heritability and environmental factors affecting vitamin D status in rural Chinese adolescent twins. J Clin Endocrinol Metab 94: 3273-3281.

5) Huh SY, Gordon CM. 2008. Vitamin D deficiency in children and adolescents: epidemiology, impact and treatment. Rev Endocr Metab Disord 9: 161-170.

6) Ovesen L, Andersen R, Jakobsen J. 2003. Geographical differences in vitamin $D$ status, with particular reference to European countries. Proc Nutr Soc 62: 813-821.

7) Valtueña J, Breidenassel C, Folle J, Gonzalez-Gross M. 2011. Retinol, beta-carotene, alpha-tocopherol and vitamin D status in European adolescents; regional differences an variability: A review. Nutr Hosp 26: 280-288.

8) Dong Y, Pollock N, Stallmann-Jorgensen IS, Gutin B, Lan L, Chen TC, Keeton D, Petty K, Holick MF, Zhu H. 2010. Low 25-hydroxyvitamin D levels in adolescents: race, season, adiposity, physical activity, and fitness. Pediatrics 125: 1104-1111.

9) Parikh SJ, Edelman M, Uwaifo GI, Freedman RJ, SemegaJanneh M, Reynolds J, Yanovski JA. 2004. The relationship between obesity and serum 1,25-dihydroxy vitamin D concentrations in healthy adults. J Clin Endocrinol Metab 89: 1196-1199.

10) Moreno LA, Gonzalez-Gross M, Kersting M, Molnar D, de Henauw S, Beghin L, Sjostrom M, Hagstromer M, Manios Y, Gilbert CC, Ortega FB, Dallongeville J, Arcella D, Warnberg J, Hallberg M, Fredriksson H, Maes L, Widhalm K, Kafatos AG, Marcos A. 2008. Assessing, understanding and modifying nutritional status, eating habits and physical activity in European adolescents: the HELENA (Healthy Lifestyle in Europe by Nutrition in Adolescence) Study. Public Health Nutr 11: 288-299.

11) Moreno LA, De Henauw S, Gonzalez-Gross M, Kersting M, Molnar D, Gottrand F, Barrios L, Sjostrom M, Manios Y, Gilbert CC, Leclercq C, Widhalm K, Kafatos A, Marcos A. 2008. Design and implementation of the Healthy Lifestyle in Europe by Nutrition in Adolescence CrossSectional Study. Int J Obes (Lond) 32 (Suppl 5): S4-11.

12) Beghin L, Castera M, Manios Y, Gilbert CC, Kersting M, De Henauw S, Kafatos A, Gottrand F, Molnar D, Sjostrom M, Leclercq C, Widhalm K, Mesana MI, Moreno LA, Libersa C. 2008. Quality assurance of ethical issues and regulatory aspects relating to good clinical practices in the HELENA Cross-Sectional Study. Int J Obes (Lond) 32 (Suppl 5): S12-18.

13) Nagy E, Vicente-Rodriguez G, Manios Y, Beghin L, Iliescu C, Censi L, Dietrich S, Ortega FB, De Vriendt T, Plada M, Moreno LA, Molnar D. 2008. Harmonization process and reliability assessment of anthropometric measurements in a multicenter study in adolescents. Int J Obes (Lond) 32 (Suppl 5): S58-65.

14) Cole TJ, Freeman JV, Preece MA. 1995. Body mass index reference curves for the UK, 1990. Arch Dis Child 73: 25-29.

15) Tanner JM, Whitehouse RH. 1976. Clinical longitudinal standards for height, weight, height velocity, weight velocity, and stages of puberty. Arch Dis Child 51: 170-179.

16) Ekelund U, Anderssen SA, Froberg K, Sardinha LB,
Andersen LB, Brage S. 2007. Independent associations of physical activity and cardiorespiratory fitness with metabolic risk factors in children: the European youth heart study. Diabetologia 50: 1832-1840.

17) Ortega FB, Artero EG, Ruiz JR, Espana-Romero V, Jimenez-Pavon D, Vicente-Rodriguez G, Moreno LA, Manios Y, Beghin L, Ottevaere C, Ciarapica D, Sarri K, Dietrich S, Blair SN, Kersting M, Molnar D, GonzalezGross M, Gutierrez A, Sjostrom M, Castillo MJ, Grp HS. 2011. Physical fitness levels among European adolescents: the HELENA study. Br J Sports Med 45: 20-29.

18) Léger LA, Mercier D, Gadoury C, Lambert J. 1988. The multistage 20 metre shuttle run test for aerobic fitness. $J$ Sports Sci 6: 93-101.

19) Brussaard JH, Lowik MRH, Steingrimsdottir L, Moller A, Kearney J, De Henauw S, Becker W, Grp E. 2002. A European food consumption survey method-conclusions and recommendations. Eur J Clin Nutr 56: S89-S94.

20) Vereecken CA, Covents M, Sichert-Hellert W, Alvira JM, Le Donne C, De Henauw S, De Vriendt T, Phillipp MK, Beghin L, Manios Y, Hallstrom L, Poortvliet E, Matthys C, Plada M, Nagy E, Moreno LA. 2008. Development and evaluation of a self-administered computerized 24-h dietary recall method for adolescents in Europe. Int $J$ Obes (Lond) 32 (Suppl 5): S26-34.

21) Haubrock J, Nothlings U, Volatier JL, Dekkers A, Ocke M, Harttig U, Illner AK, Knuppel S, Andersen LF, Boeing H. 2011. Estimating usual food intake distributions by using the multiple source method in the EPIC-potsdam calibration study. J Nutr 141: 914-920.

22) Black AE. 2000. Critical evaluation of energy intake using the Goldberg cut-off for energy intake: basal metabolic rate. A practical guide to its calculation, use and limitations. Int J Obes (Lond) 24: 1119-1130.

23) Iliescu C, Beghin L, Maes L, De Bourdeaudhuij I, Libersa C, Vereecken C, Gonzalez-Gross M, Kersting M, Molnar D, Leclercq C, Sjostrom M, Manios Y, Wildhalm K, Kafatos A, Moreno LA, Gottrand F. 2008. Socioeconomic questionnaire and clinical assessment in the HELENA Cross-Sectional Study: methodology. Int J Obes (Lond) $\mathbf{3 2}$ (Suppl 5): S19-25.

24) Currie CE, Elton RA, Todd J, Platt S. 1997. Indicators of socioeconomic status for adolescents: the WHO Health Behaviour in School-aged Children Survey. Health Educ Res 12: 385-397.

25) Gonzalez-Gross M, Breidenassel C, Gomez-Martinez S, Ferrari M, Beghin L, Spinneker A, Diaz LE, Maiani G, Demailly A, Al-Tahan J, Albers U, Warnberg J, StoffelWagner B, Jimenez-Pavon D, Libersa C, Pietrzik K, Marcos A, Stehle P. 2008. Sampling and processing of fresh blood samples within a European multicenter nutritional study: evaluation of biomarker stability during transport and storage. Int J Obes (Lond) 32 (Suppl 5): S66-75.

26) FNB, IOM. 2011. Dietary Reference Intakes for Calcium, Phosphorus, Magnesium, Vitamin D and Fluoride. National Academy Press, Washington, DC.

27) Racinais S, Hamilton B, Li CK, Grantham J. 2010. Vitamin D and physical fitness in Qatari girls. Arch Dis Child 95: 854-855.

28) Penckofer S, Kouba J, Byrn M, Estwing Ferrans C. 2010. Vitamin D and depression: where is all the sunshine? Issues Ment Health Nurs 31: 385-393.

29) Holick MF. 2005. The vitamin D epidemic and its health consequences. J Nutr 135: 2739S-2748S. 
30) Moreno LA, Valtueña J, Perez-Lopez F, Gonzalez-Gross M. 2011. Health effects related to low vitamin D concentrations: beyond bone metabolism. Ann Nutr Metab 59: $22-27$.

31) Absoud M, Cummins C, Lim MJ, Wassmer E, Shaw N. 2011. Prevalence and predictors of vitamin D insufficiency in children: a Great Britain population based study. PLoS One 6: e22179.

32) Bener A, Al-Ali M, Hoffmann GF. 2009. Vitamin D deficiency in healthy children in a sunny country: associated factors. Int J Food Sci Nutr 60 (Suppl 5): 60-70.

33) Hill TR, Cotter AA, Mitchell S, Boreham CA, Dubitzky W, Murray L, Strain JJ, Flynn A, Robson PJ, Wallace JM, Kiely M, Cashman KD. 2008. Vitamin D status and its determinants in adolescents from the Northern Ireland Young Hearts 2000 cohort. Br J Nutr 99: 1061-1067.

34) Lehtonen-Veromaa M, Mottonen T, Irjala K, Karkkainen M, Lamberg-Allardt C, Hakola P, Viikari J. 1999. Vitamin D intake is low and hypovitaminosis D common in healthy 9- to 15-year-old Finnish girls. Eur J Clin Nutr 53: 746-751.

35) Guillemant J, Le HT, Maria A, Allemandou A, Peres G, Guillemant S. 2001. Wintertime vitamin D deficiency in male adolescents: Effect on parathyroid function and response to vitamin D-3 supplements. Osteoporos Int 12 $875-879$.

36) Gregory Jr LS, Bates CJ, Prentice A, Jackson LV, Smithers G, Wenlock R, Farron H (ed). 2000. National Diet and Nutrition Survey: Young People Aged 4 to 18 Years. HMSO, London.

37) Lanham-New SA, Thompson RL, More J, Brooke-Wavell K, Hunking P, Medici E. 2007. Importance of vitamin $\mathrm{D}$, calcium and exercise to bone health with specific reference to children and adolescents. Nutr Bull 32: 364-377.

38) Lenders CM, Feldman HA, Von Scheven E, Merewood A, Sweeney C, Wilson DM, Lee PD, Abrams SH, Gitelman SE, Wertz MS, Klish WJ, Taylor GA, Chen TC, Holick MF. 2009. Relation of body fat indexes to vitamin D status and deficiency among obese adolescents. Am J Clin Nutr 90: $459-467$.
39) Liel Y, Ulmer E, Shary J, Hollis BW, Bell NH. 1988. Low circulating vitamin-D in obesity. Calcif Tissue Int 43: 199-201.

40) Weng FL, Shults J, Leonard MB, Stallings VA, Zemel BS. 2007. Risk factors for low serum 25-hydroxyvitamin D concentrations in otherwise healthy children and adolescents. Am J Clin Nutr 86: 150-158.

41) Jago R, Harrell JS, McMurray RG, Edelstein S, El Ghormli L, Bassin S. 2006. Prevalence of abnormal lipid and blood pressure values among an ethnically diverse population of eighth-grade adolescents and screening implications. Pediatrics 117: 2065-2073.

42) Foo LH, Zhang Q, Zhu K, Ma G, Hu X, Greenfield H, Fraser DR. 2009. Low vitamin D status has an adverse influence on bone mass, bone turnover, and muscle strength in Chinese adolescent girls. J Nutr 139: 1002-1007.

43) Holick MF. 2009. Vitamin D: the other steroid hormone for muscle function and strength. Menopause 16: 1077-1078.

44) Garaulet M, Ortega FB, Ruiz JR, Rey-Lopez JP, Beghin L, Manios Y, Cuenca-Garcia M, Plada M, Diethelm K, Kafatos A, Molnar D, Al-Tahan J, Moreno LA. 2011. Short sleep duration is associated with increased obesity markers in European adolescents: effect of physical activity and dietary habits. The HELENA study. Int J Obes (Lond) 35: 1308-1317.

45) Guillemant J, Allemandou A, Peres G, Guillemant SE. 2000. Wintertime vitamin D deficiency in male adolescents: Response to vitamin D3 supplements. J Bone Miner Res 15: S450.

46) Tylavsky FA, Cheng SL, Lyytikainen A, Viljakainen H, Lamberg-Allardt C. 2006. Strategies to improve vitamin D status in northern European children: Exploring the merits of vitamin D fortification and supplementation. J Nutr 136: 1130-1134.

47) Zemel MB. 2002. Regulation of adiposity and obesity risk by dietary calcium. Bull Int Dairy Feder 135: 103-105.

48) Heaney RP, Davies KM, Barger-Lux MJ. 2002. Calcium and weight: Clinical studies. J Am Coll Nutr 21: $152 \mathrm{~s}-155 \mathrm{~s}$. 